\title{
Riqueza, composición y diversidad de la comunidad de moluscos asociada al sustrato rocoso intermareal de playa Parque de la Reina, Acapulco, México
}

Community structure, richness, and diversity of mollusks associated on the intertidal rocky substrate at Parque de la Reina Beach, Acapulco, Mexico

\section{Carmina Torreblanca-Ramírez ${ }^{1}$, Rafael Flores-Garza², Pedro Flores-Rodríguez², Sergio García-Ibáñez ${ }^{2}$ y Lizeth Galeana-Rebolledo ${ }^{2}$}

\begin{abstract}
${ }^{1}$ Doctorado en Ciencias Ambientales, Unidad de Ciencias de Desarrollo Regional, Universidad Autónoma de Guerrero, Calle Pino s/n Colonia El Roble Acapulco, Gro. C. P. 39640, México. carminatorreblanca@yahoo.com.mx

${ }^{2}$ Unidad Académica de Ecología Marina, Laboratorio de Ecología Costera y Sustentabilidad, Universidad Autónoma de Guerrero, Av. Gran Vía Tropical Nº 20, Fraccionamiento Las Playas, Acapulco, Guerrero, CP. 39390, México
\end{abstract}

\begin{abstract}
The information available about marine biodiversity in the State of Guerrero, Mexico, is scarce as designated by the National Commission for the Conservation and Use of Biodiversity. To gain on this issue, this work was carried on at the rocky intertidal zone of Parque de la Reina beach, Acapulco, which is located in the Priority Region for Marine Conservation and Biodiversity Use Number 32 . The objective was to establish the mollusks species richness, community structure, determine the density and degree of species occurrence, composition by size and diversity. The sampling area was $10 \mathrm{~m}^{-2}$ and the sampling unit was $1 \mathrm{~m}^{-2}$. Fifty eight species were identified, with 4 new records for the intertidal rocky shores of Acapulco. The best represented families were: Muricidae, Ischnochitonidae, and Mytilidae. The observed density was of $207.55 \mathrm{~m}^{-2}$. Twenty one species were rated as dominant. Macrocypraea cervinetta had the highest size. The mollusks richness was high, as is expected in the tropics. The constant and dominant species accounted for $55.16 \%$ of the total, and considered as representative of the community. The density found was higher than has been previously reported for the State of Guerrero; the type of substrate is one of the determining factors defining species richness and abundance.
\end{abstract}

Key words: Check list, degree of occurrence, dominance, size and records

\begin{abstract}
Resumen.- El conocimiento de la biodiversidad marina en el Estado de Guerrero, México es escaso, lo que es señalado por la Comisión Nacional para la Conservación y Uso de la Biodiversidad. Para avanzar en este conocimiento, se trabajó en el intermareal rocoso de Playa Parque de la Reina, Acapulco, que se encuentra ubicada en la región marina prioritaria para la conservación y uso de la biodiversidad número 32. El objetivo fue determinar la riqueza de especies de moluscos, conocer la composición de la comunidad, la densidad, el grado de aparición de las especies, la composición por tallas y la diversidad. El área de muestreo fue de $10 \mathrm{~m}^{-2}$ y la unidad de muestreo fue $1 \mathrm{~m}^{-2}$. Se identificaron 58 especies, 4 nuevos registros para el intermareal rocoso de Acapulco. Las familias más representadas fueron: Muricidae, Ischnochitonidae, y Mytilidae. La densidad fue de 207,55 organismos $\mathrm{m}^{-2}$. Fueron dominantes 21 especies. Macrocypraea cervinetta presentó la mayor talla. La riqueza de moluscos fue alta, coincidiendo con lo esperado en una zona tropical. Las especies dominantes y constantes representaron el 55,16\%, y se consideran representativas de la comunidad. La densidad encontrada es la más alta que se ha reportado para Guerrero, México y el tipo de sustrato es uno de los factores determinantes que define la riqueza de especies y la abundancia.
\end{abstract}

Palabras clave: Inventario, grado de aparición, dominancia, tallas y registros

\section{INTRODUCCIÓN}

En México, han sido delimitadas y caracterizadas por la Comisión Nacional para la Conservación y Uso de la Biodiversidad (CONABIO) 70 áreas costeras y oceánicas consideradas prioritarias por su alta diversidad biológica, por el uso de sus recursos y por su falta de conocimiento sobre biodiversidad. El área 32 (Región Marina Prioritaria $\mathrm{N}^{\circ}$ 32) se encuentra ubicada en el Estado de Guerrero y se conoce como área de Coyuca-Tres Palos, tiene una extensión de $829 \mathrm{~km}^{2}$. De acuerdo a la CONABIO, las problemáticas que presenta esta área son: la modificación 
de su entorno por descargas de agua dulce, agroquímicos y fertilizantes, desechos ganaderos; daño al ambiente por el transporte turístico; usos de recursos como las especies de aves en riesgo; especies introducidas como la tilapia y palma cocotera; desconocimiento de la normativa vigente para el aprovechamiento de los recursos. Respecto a la conservación, la CONABIO señala el escaso conocimiento de la región (Arriaga et al. 1998).

El intermareal rocoso es uno de los hábitats que se encuentra en la región marina prioritaria 32 y es considerado como uno de los más diversos del ambiente marino (Tait \& Dipper 1998); éste se ubica en la costa que corresponde al municipio de Acapulco y en Playa Parque de la Reina.

Con respecto a los moluscos, la CONABIO ha señalado que es un grupo sobre el cual se tiene poca información y que se requiere de mayor investigación en toda la República Mexicana. Dentro de la información necesaria se encuentran el análisis de la riqueza y diversidad de las especies que conforman la comunidad, además conocer el estado de las poblaciones y de la comunidad, siendo esencial para que se desarrolle una administración adecuada de los recursos y prevenir los riesgos que conducen al agotamiento o sobreexplotación de los mismos.

La mayoría de los trabajos publicados sobre los moluscos del litoral del Pacífico Mexicano son de carácter faunístico o taxonómico; algunos tratan aspectos de diversidad y variación a través del tiempo (Stuardo \& Villarroel 1976, Baqueiro \& Stuardo 1977, Baqueiro 1979, Reguero \& García 1989, Holguín \& González 1989, 1994, Landa \& Arciniega 1998, Olabarría 1999, Villarroel et al. 2000, Ríos-Jara et al. 2001, Flores-Campaña et al. 2007 y Landa-Jaime et al. 2007) y otros han investigado acerca de la distribución y abundancia (Román et al. 1991, Esqueda et al. 2000, García \& Álvarez 2007, Ortíz-Arellano \& Flores-Campaña 2008, Flores-Rodríguez et al. 2010, Reyes-Gómez et al. 2010).

Para el Estado de Guerrero, hay trabajos que presentan resultados sobre el análisis de la comunidad de los moluscos en el intermareal rocoso de distintos sitios que se ubican a lo largo de la costa (Flores 2004). También existen informes de inventarios de especies como en Zihuatanejo (Salcedo et al. 1988).

Para Acapulco, existen investigaciones sobre moluscos del intermareal rocoso, respecto a la riqueza de especies, zonación, composición de las poblaciones y comunidades y otros aspectos ecológicos (Villalpando 1986, Delgado 1989, García 1994, Flores-Rodríguez et al. 2003, 2007, Valdez-González et al. 2004, Flores-Garza et al. 2007, 2010, 2011, Barba-Marino et al. 2010).

Este estudio se llevó a cabo en el intermareal rocoso de Playa Parque de la Reina, Acapulco y sus objetivos fueron: 1) determinar la riqueza de especies de las Clases Gastropoda, Bivalvia y Polyplacophora, 2) conocer la composición de la comunidad a partir de la representación de clases y familias con base en: a) riqueza de especies, b) abundancia, 3) estimar la densidad de las poblaciones que integran la comunidad de moluscos, 4) analizar el grado de aparición las especies, 5) examinar la estructura de tallas de las poblaciones y 6) estimar la diversidad malacológica del ambiente intermareal.

\section{Materiales y MÉtodos}

\section{Área de MUESTReo}

Parque de la Reina es una pequeña playa del puerto de Acapulco, que se ubica entre $16^{\circ} 50^{\prime} 58,27^{\prime \prime} \mathrm{N}$ y 9954'01,85' 'W. La playa mide aproximadamente $66,74 \mathrm{~m}$ de largo, con acumulación artificial de rocas de tamaños variables y consistencia porosa, presenta escombros caracterizados por restos de pisos y columnas de concreto y la intensidad del oleaje es media.

\section{Trabajo en terreno}

Se llevaron a cabo 3 muestreos en el intermareal rocoso, un exploratorio en enero del 2009 y dos formales en abril y mayo del mismo año. El muestreo se realizó durante las horas de marea baja y los días de luna nueva. El área de muestreo fue de $10 \mathrm{~m}^{-2} \mathrm{y}$ la unidad de muestreo fue de $1 \mathrm{~m}^{-2}$. El muestreo fue aleatorio y luego una cuerda de 30 $\mathrm{m}$ de longitud se colocó en paralelo a la costa, en la zona intermareal. Para delimitar la unidad de muestreo se utilizó un marco de forma cuadrada de $1 \mathrm{~m}$ x $1 \mathrm{~m}$, de tubos de PVC. El marco que delimita la unidad de muestreo se colocó en el punto seleccionado. Los individuos de moluscos encontrados vivos dentro de la unidad de muestreo fueron recolectados y colocados dentro de un recipiente de plástico que contenía agua de mar y se protegió de la luz solar. Después de concluir la recolecta de la primera unidad de muestreo, se dejó un espacio de $2 \mathrm{~m}$ siguiendo la cuerda y se colocó el marco para delimitar el siguiente $\mathrm{m}^{2}$ donde se recolectaría la muestra. Este procedimiento se repitió hasta completar $10 \mathrm{~m}^{2}$. Al terminar el muestreo se procedió a la preservación, colocando a los ejemplares en frascos 
con alcohol etílico al 96\% y posteriormente el material fue trasladado al laboratorio. Los especímenes fueron identificados y cuantificados, utilizando literatura especializada (Keen 1971, Kaas \& Van Belle 1985, 1990, 1994, Coan \& Valentich-Scott 2012) y la nomenclatura se actualizó de acuerdo a Skoglund $(2001,2002)$ para Gastropoda y Bivalvia, y Sirenko (2006) para Polyplacophora. Los ejemplares recolectados fueron medidos en longitud (mm), para lo cual, se utilizó un calibrador digital. Posterior a la identificación y medición de los organismos, estos se depositaron en la Colección Malacológica de la Unidad Académica de Ecología Marina, Universidad Autónoma de Guerrero y en la Colección Nacional de Moluscos en la Universidad Nacional Autónoma de México.

\section{Análisis de datos}

La riqueza se consideró como el número de especies de moluscos presentes. La composición de la comunidad se analizó con base en el número de especies y la abundancia relativa que representan a cada clase y a cada familia. La densidad se evaluó en organismos $\mathrm{m}^{-2}$. El grado de aparición de las especies (ubicación jerárquica) dentro de la comunidad, se determinó usando el método de correlación de Olmstead-Tukey, representado mediante gráficas de cuadrantes (Sokal \& Rohlf 1969), que tuvo como base el cálculo de 2 estimadores, el primero fue el promedio de la abundancia relativa de todas las especies, expresado en logaritmo de la abundancia $+1 \mathrm{y}$ el segundo estimador, fue el promedio del número de muestras en la cual todas las especies están presentes (frecuencia de ocurrencia), expresado en porcentaje. Con el cálculo de estos estimadores se analizó gráficamente el porcentaje de la frecuencia de aparición de cada especie contra el porcentaje de la abundancia relativa promedio de cada especie, expresada en el logaritmo de la abundancia +1 . Las especies dominantes fueron aquellas cuyos valores de abundancia y frecuencia de ocurrencia sobrepasan la media aritmética de ambos valores estimados. Las especies constantes fueron aquellas cuyo valor de abundancia no sobrepasan el valor promedio de la abundancia total, pero si sobrepasan el valor promedio estimado para la frecuencia de ocurrencia. Las especies numerosas poco frecuentes se caracterizaron porque su valor de abundancia es mayor al valor promedio estimado para la abundancia y el valor de frecuencia de ocurrencia no rebasa el valor promedio estimado para esta variable. Las especies ocasionales fueron aquellas que el valor de su abundancia y frecuencia de ocurrencia no sobrepasan a la media aritmética estimada para frecuencia de ocurrencia y abundancia. El análisis de la estructura en talla de las poblaciones que componen la comunidad de moluscos se realizó en relación a sus longitudes, obteniendo los valores de los estadísticos descriptivos como máximos, mínimos, promedios y desviación estándar. Estos valores se expresaron en mm. La diversidad se midió aplicando el índice de Shannon-Wiener (H’) y el índice de uniformidad o equidad de Pielou (J').

\section{Resultados}

Se analizaron 4151 organismos y se identificaron 58 especies de moluscos que componen la comunidad asociada al sustrato intermareal rocoso, distribuidas en 37 especies de Gastropoda (63,79\%), 8 de Bivalvia (13,79\%) y 13 de Polyplacophora (22,41\%). La Clase con mayor abundancia relativa fue Gastropoda, que presentó el 67,83\% de los individuos representados en la muestra, seguida por Polyplacophora con 31,53\% y finalmente Bivalvia con 0,62\% de la abundancia (Fig. 1).

En cuanto a la composición de la comunidad por familias, se identificaron 25 familias, de las cuales 16 corresponden a la Clase Gastropoda, 4 Bivalvia y 5 Polyplacophora.

Referente a la composición de la clase Gastropoda con base en la representación por familias, las familias mejor representadas en riqueza de especies fueron: Muricidae (7 especies), Columbellidae (6 especies) y Calyptraeidae (4 especies) (Tabla 1) y la mejor representación en cuanto a su abundancia relativa la presentó la familia Muricidae con el 20,23\%. En la Clase Bivalvia la familia mejor representada en cuanto a riqueza fue Mytilidae (4 especies) (Tabla 2) y en abundancia relativa fue Chamidae (0,26\%). En la Clase Polyplacophora las familias mejor representadas en cuanto a riqueza fueron: Ischnochitonidae (3 especies), Chaetopleuridae con (3 especies), Chitonidae con (3 especies) (Tabla 3) y en abundancia relativa fue Chitonidae (27,63\%).

Para el Estado de Guerrero las especies Mitrella xenia (Dall, 1919), Macrocypraea cervinetta (Kiener, 1843) y Rhinoclavis (Ochetoclava) gemmata (Hinds, 1844) de la clase Gastropoda y Brachidontes puntarenensis (Pilsbry \& Lowe, 1932) de la Clase Bivalvia se consideran nuevos registros (Fig. 2, 3).

Se estimó una densidad de 207,55 organismos $\mathrm{m}^{-2}$, de los cuales 140,8 organismos $\mathrm{m}^{-2}$ correspondieron a la Clase Gastropoda, 1,3 organismos $\mathrm{m}^{-2}$ a Bivalvia y 65,45 
Tabla 1. Riqueza de especies, familia, grado de aparición, densidad (organismos $\mathrm{m}^{-2}$ ) y longitudes (mm) de las especies de la Clase Gastropoda asociadas al sustrato rocoso intermareal de playa Parque de la Reina, Acapulco, Guerrero, México / Species richness, family, degree of species occurrence, density (organisms $\mathrm{m}^{-2}$ ) and sizes $(\mathrm{mm})$ of the Class Gastropoda species associated with intertidal rocky substrate at Parque de la Reina Beach, Acapulco, Guerrero, Mexico

\begin{tabular}{|c|c|c|c|c|c|c|c|}
\hline \multirow[b]{2}{*}{ Especies / Familias } & \multirow[b]{2}{*}{$\mathrm{n}$} & \multirow[b]{2}{*}{$\mathrm{Ga}$} & \multirow[b]{2}{*}{$\mathrm{De}$} & \multicolumn{4}{|c|}{ Longitud (mm) } \\
\hline & & & & Min. & Max. & Ave. & Ds \\
\hline \multicolumn{8}{|l|}{ LOTTIIDAE } \\
\hline Tectura fascicularis (Menke, 1851) & 250 & D & 12,50 & 5,00 & 30,34 & 15,05 & 5,30 \\
\hline Scurria mesoleuca (Menke, 1851) & 3 & $\mathrm{O}$ & 0,15 & 24,24 & 25,85 & 24,81 & 0,89 \\
\hline \multicolumn{8}{|l|}{ TROCHIDAE } \\
\hline Tegula (Agathistoma) globulus (Carpenter, 1857) & 843 & $\mathrm{D}$ & 42,15 & 2,92 & 9,87 & 6,69 & 1,27 \\
\hline \multicolumn{8}{|l|}{ TURBINIDAE } \\
\hline Astraea (Uvanilla) buschii (Philippi, 1844) & 5 & $\mathrm{O}$ & 0,25 & 6,04 & 10,38 & 7,43 & 1,7 \\
\hline \multicolumn{8}{|l|}{ NERITIDAE } \\
\hline Nerita (Cymostyla) scabricosta Lamarck, 1822 & 1 & $\mathrm{O}$ & 0,05 & 14,05 & 14,05 & 14,05 & 0 \\
\hline Nerita (Theliostyla) funiculata Menke, 1851 & 1 & $\mathrm{O}$ & 0,05 & 4,90 & 4,90 & 4,90 & 0 \\
\hline \multicolumn{8}{|l|}{ LITTORINIDAE } \\
\hline Nodilittorina (Nodilittorina) aspera (Philippi 1846) & 58 & $\mathrm{D}$ & 2,90 & 2,52 & 9,19 & 4,89 & 1,43 \\
\hline \multicolumn{8}{|l|}{ CERITHIIDAE } \\
\hline Rhinoclavis (Ochetoclava) gemmata (Hinds, 1844)* & 2 & $\mathrm{C}$ & 0,10 & 10,61 & 11,83 & 11,22 & 0,86 \\
\hline \multicolumn{8}{|l|}{ PLANAXIDAE } \\
\hline Planaxis obsoletus Menke, 1851 & 19 & D & 0,95 & 5,28 & 14,70 & 11,09 & 2,82 \\
\hline \multicolumn{8}{|l|}{ EPITONIIDAE } \\
\hline Opalia (Nodiscala) infrequens (Adams, 1852) & 39 & NPF & 1,95 & 3,90 & 7,07 & 6,00 & 0,64 \\
\hline \multicolumn{8}{|l|}{ CALYPTRAEIDAE } \\
\hline Crepidula aculeata (Gmelin, 1791) & 1 & $\mathrm{O}$ & 0,05 & 5,16 & 5,16 & 5,16 & 0 \\
\hline Crepidula incurva (Broderip, 1834) & 2 & $\mathrm{O}$ & 0,10 & 5,88 & 6,75 & 6,31 & 0,61 \\
\hline Crucibulum (Crucibulum) scutellatum (Wood, 1828) & 1 & $\mathrm{O}$ & 0,05 & 14,33 & 14,33 & 14,33 & 0 \\
\hline Crucibulum (Crucibulum) umbrella (Deshayes, 1830) & 14 & D & 0,70 & 5,05 & 49,14 & 39,98 & 10,63 \\
\hline \multicolumn{8}{|l|}{ CYPRAEIDAE } \\
\hline Macrocypraea cervinetta (Kiener, 1843)* & 5 & $\mathrm{C}$ & 0,25 & 8,55 & 65,15 & 36,69 & 24,73 \\
\hline Mauritia arabicula (Lamarck, 1811) & 8 & $\mathrm{C}$ & 0,40 & 19,02 & 26,95 & 21,70 & 2,54 \\
\hline Jenneria pustulata [Lightfoot, 1786] & 1 & $\mathrm{O}$ & 0,05 & 3,76 & 3,76 & 3,76 & 0 \\
\hline \multicolumn{8}{|l|}{ MURICIDAE } \\
\hline Muricopsis (Muricopsis) zeteki Hertlein \& Strong 1951 & 30 & NPF & 1,50 & 12,29 & 15,39 & 13,83 & 1,55 \\
\hline Trachypollia lugubris (C.B. Adams, 1852) & 88 & $\mathrm{D}$ & 4,40 & 7,95 & 23,20 & 15,63 & 4,16 \\
\hline Mancinella speciosa (Valenciennes, 1832) & 26 & NPF & 1,30 & 7,49 & 25,93 & 13,57 & 4,71 \\
\hline Mancinella triangularis (Blainville, 1832) & 356 & D & 17,80 & 3,21 & 14,10 & 8,27 & 2,03 \\
\hline Plicopurpura pansa (Gould, 1853) & 2 & $\mathrm{C}$ & 0,10 & 14,20 & 21,40 & 17,80 & 5,09 \\
\hline Stramonita biserialis (Blainville, 1832) & 175 & D & 8,75 & 4,44 & 22,22 & 11,25 & 4,24 \\
\hline Vasula melones (Duclos, 1832) & 1 & $\mathrm{O}$ & 0,05 & 39,15 & 39,15 & 39,15 & 0 \\
\hline \multicolumn{8}{|l|}{ BUCCINIDAE } \\
\hline Cantharus (Pollia) sanguinolentus (Duclos, 1833) & 7 & $\mathrm{C}$ & 0,35 & 13,32 & 22,10 & 16,92 & 2,73 \\
\hline Engina tabogaensis Bartsch, 1931 & 1 & $\mathrm{O}$ & 0,05 & 8,60 & 8,60 & 8,60 & 0 \\
\hline \multicolumn{8}{|l|}{ COLUMBELLIDAE } \\
\hline Columbella fuscata Sowerby, 1832 & 172 & D & 8,60 & 7,07 & 27,24 & 16,51 & 2,20 \\
\hline Columbella major Sowerby, 1832 & 17 & NPF & 0,85 & 16,31 & 23,53 & 20,72 & 1,65 \\
\hline Costoanachis nigrofusca (Carpenter, 1857) & 373 & DO & 18,65 & 2,97 & 8,32 & 6,53 & 0,90 \\
\hline Parvanachis dalli (Bartsch, 1931) & 4 & $\mathrm{C}$ & 0,20 & 3,91 & 6,33 & 5,59 & 1,12 \\
\hline Mitrella ocellata (Gmelin, 1791) & 177 & $\mathrm{D}$ & 8,85 & 3,22 & 12,12 & 10,46 & 1,05 \\
\hline Mitrella xenia (Dall, 1919)* & 97 & D & 4,85 & 4,70 & 14,49 & 7,92 & 1,61 \\
\hline FASCIOLARIIDAE & & & & & & & \\
\hline Leucozonia cerata (Wood, 1828) & 5 & $\mathrm{C}$ & 0,25 & 17,17 & 30,02 & 21,90 & 4,90 \\
\hline Opeatostoma pseudodon (Burrow, 1815) & 18 & D & 0,90 & 18,32 & 31,60 & 25,10 & 3,95 \\
\hline MITRIDAE & & & & & & & \\
\hline Mitra (Strigatella) tristis Broderip, 1836 & 2 & $\mathrm{C}$ & 0,10 & 10,75 & 14,54 & 12,64 & 2,67 \\
\hline SIPHONARIIDAE & & & & & & & \\
\hline Siphonaria (Heterosiphonaria) maura Sowerby, 1835 & 5 & $\mathrm{o}$ & 0,25 & 8,49 & 10,82 & 9,79 & 0,99 \\
\hline Siphonaria (Heterosiphonaria) palmata Carpenter, 1857 & 7 & $\mathrm{O}$ & 0,35 & 11,71 & 15,72 & 13,13 & 1,33 \\
\hline
\end{tabular}

*= No registros previos; $\mathrm{n}=$ número de organismos analizados; $\mathrm{Ga}=$, Grado de aparición $(\mathrm{D}=$ Dominante, $\mathrm{C}=\mathrm{Común}$, $\mathrm{NPF}=$ Numeroso poco frecuente, $\mathrm{O}=$ Ocasional); $\mathrm{De}=$ densidad; $\mathrm{Ave}=$ media, $\mathrm{Ds}=$ desviación estándar 


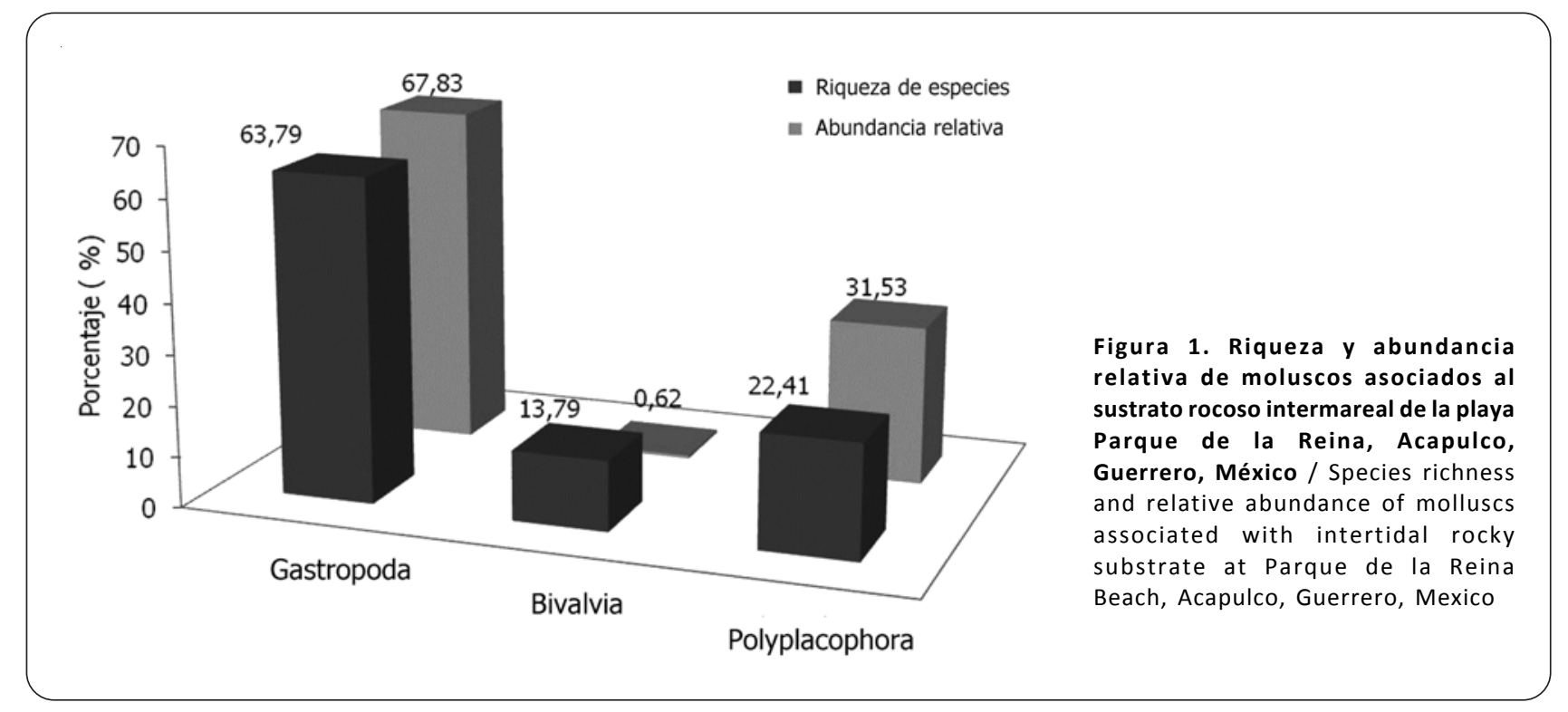

Tabla 2. Riqueza, representación por Familia, grado de aparición, densidad (organismos $\mathrm{m}^{-2}$ ) y tallas $(\mathrm{mm}) \mathrm{de}$ las especies de la Clase Bivalvia asociadas al sustrato rocoso intermareal de playa Parque de la Reina, Acapulco, Guerrero, México / Species richness, Family, degree of species occurrence, density (organisms $\mathrm{m}^{-2}$ ) and sizes $(\mathrm{mm})$ of the Class Bivalvia species associated with intertidal rocky substrate at Parque de la Reina Beach, Acapulco, Guerrero, Mexico

\begin{tabular}{|c|c|c|c|c|c|c|c|}
\hline \multirow{2}{*}{ Especies / Familias } & \multirow{2}{*}{$\mathrm{n}$} & \multirow{2}{*}{$\mathrm{Ga}$} & \multirow{2}{*}{$\mathrm{De}$} & \multicolumn{4}{|c|}{ Longitud (mm) } \\
\hline & & & & Min. & Max. & Ave. & Ds \\
\hline \multicolumn{8}{|l|}{ MYTILIDAE } \\
\hline Brachidontes adamsianus (Dunker, 1857) & 2 & $\mathrm{O}$ & 0,10 & 4,55 & 4,94 & 4,74 & 0,27 \\
\hline Brachidontes puntarenensis (Pilsbry \& Lowe, 1932)* & 4 & $\mathrm{D}$ & 0,20 & 4,93 & 5,97 & 5,56 & 0,44 \\
\hline Modiolus (Modiolus) capax (Conrad, 1837) & 1 & $\mathrm{O}$ & 0,05 & 31,13 & 31,13 & 31,13 & 0 \\
\hline Lithophaga (Myoforceps) aristata (Dillwyn, 1817) & 1 & $\mathrm{O}$ & 0,05 & 7,50 & 7,50 & 7,50 & 0 \\
\hline \multicolumn{8}{|l|}{ ISOGNOMONIDAE } \\
\hline Isognomon (Melina) janus (Carpenter, 1857) & 1 & $\mathrm{O}$ & 0,05 & 12,19 & 11,14 & 11,66 & 0,74 \\
\hline \multicolumn{8}{|l|}{ CARDITIDAE } \\
\hline Cardites grayi (Dall, 1903) & 6 & $\mathrm{D}$ & 0,30 & 9,60 & 30,40 & 22,53 & 8,15 \\
\hline \multicolumn{8}{|l|}{ CHAMIDAE } \\
\hline Chama (Chama) corallina Olsson, 1971 & 8 & $\mathrm{D}$ & 0,40 & 24,03 & 48,89 & 35,92 & 7,85 \\
\hline Chama (Chama) mexicana Carpenter, 1857 & 3 & NPF & 0,15 & 20,21 & 33,61 & 26,33 & 6,77 \\
\hline
\end{tabular}

*=No registros previos; $\mathrm{n}=$ número de organismos analizados; $\mathrm{Ga}=$ Grado de aparición $(\mathrm{D}=$ Dominante, $\mathrm{C}=\mathrm{Común}$, $\mathrm{NPF}=$ Numeroso poco frecuente, $\mathrm{O}=$ Ocasional); $\mathrm{De}=$ densidad; Ave=media, Ds=desviación estándar 


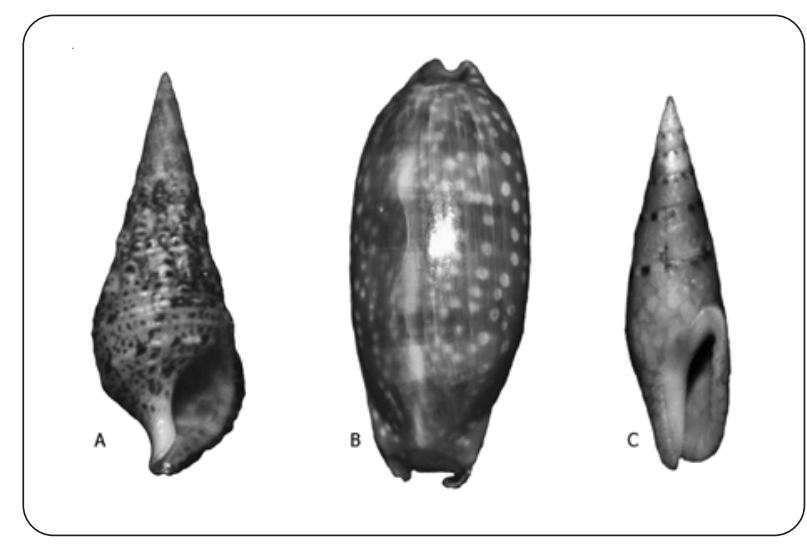

Figura 2. Nuevos registros de la Clase Gastropoda para el intermareal rocoso del Acapulco: A) Rhinoclavis (O.) gemmata (Hinds, 1844), B) Macrocypraea cervinetta (Kiener, 1843) y C) Mitrella xenia (Dall, 1919) / New records of the Class Gastropoda to the rocky intertidal zone of Acapulco: A) Rhinoclavis (O.) gemmata (Hinds, 1844), B) Macrocypraea cervinetta (Kiener, 1843) y C) Mitrella xenia (Dall, 1919)

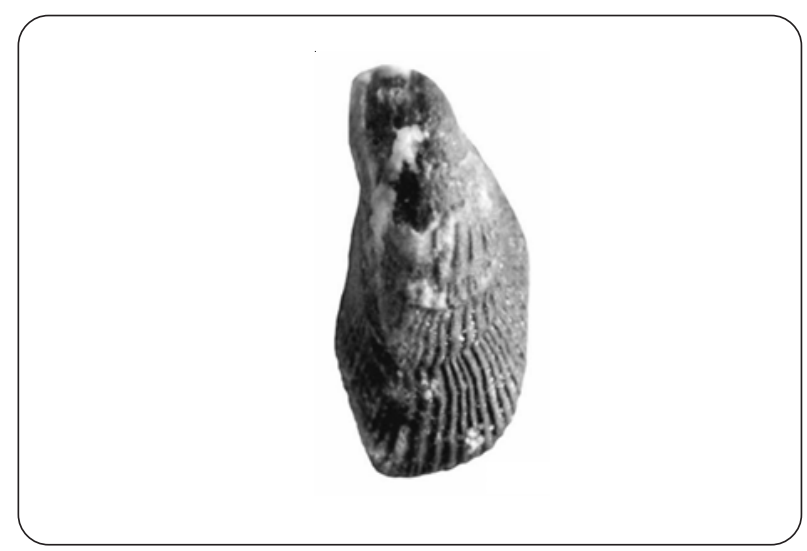

Figura 3. Nuevo registro de la Clase Bivalvia para el intermareal rocoso del Acapulco: Brachidontes puntarenensis (Pilsbry \& Lowe, 1932) / New record of the Class Bivalvia to the rocky intertidal zone of Acapulco Brachidontes puntarenensis (Pilsbry \& Lowe, 1932)

organismos $\mathrm{m}^{-2}$ a Polyplacophora. Las especies que presentaron mayor densidad en Gastropoda fueron Tegula (Agathistoma) globulus (Carpenter, 1857) $(42,15$ organismos $\mathrm{m}^{-2}$ ), Costoanachis nigrofusca (Carpenter, 1857) (18,65 organismos $\left.\mathrm{m}^{-2}\right)$ y Mancinella triangularis (Blainville, 1832) (17,8 organismos $\left.\mathrm{m}^{-2}\right)$ (Tabla 1); y en Polyplacophora Chiton (Chiton) articulatus Sowerby, 1832 (56,2 organismos $\mathrm{m}^{-2}$ ), seguido por Ischnochiton (Ischnochiton) muscarius (Reeve, 1847) (4,2 organismos $\mathrm{m}^{-2}$ ) (Tabla 3).

El grado de aparición de las especies las especies en la comunidad mostró que del total de las especies representadas en las muestras, 21 fueron dominantes (14 Gastropoda, 4 Polyplacophora y 3 Bivalvia), 11 constantes (7 Gastropoda y 4 Polyplacophora), 5 numerosas poco frecuentes (4 Gastropoda y 1 Bivalvia) y 21 ocasionales (12 Gastropoda, 5 Polyplacophora y 4 Bivalvia). Este mismo análisis hecho para cada una de las Clases por separado, encontró que el 37,83\% de las especies de Gastropoda resultaron dominantes siendo esta Clase la que porcentualmente presentó más especies dominantes, seguida por Bivalvia con el 33,33\% y Polyplacophora con 30,70\% (Fig. 4).

En relación a la longitud en Gastropoda, Macrocypraea cervinetta fue la especie que registró la mayor longitud con 61,15 mm (mínimo = 8,55 mm; media \pm desviación estándar 36,69 $\pm 24,73 \mathrm{~mm}$ ) y Jenneria pustulata (Lightfoot, 1786) la de menor longitud con 3,76 mm. En Bivalvia, la especie que presentó la mayor longitud fue Chama (Chama) corallina Olsson, 1971 con 48,89 mm (mínimo = 24,03 mm; 35,92 \pm 7,85 mm) y Brachidontes adamsianus (Dunker, 1857) fue la de menor longitud con 4,94 mm. En Polyplacophora, Chiton (Chiton) articulatus Sowerby, 1832 presentó la mayor longitud con 42,06 mm (mínimo = 4,73 mm; 21,66 $\pm 1,07 \mathrm{~mm}$ ) y Callistoplax retusa (Sowerby in Broderip \& Sowerby, 1832) la menor longitud con $4,35 \mathrm{~mm}$.

El índice de diversidad de Shannon-Wiener (H') calculado para las especies de las 3 clases en conjunto, fue de $\mathrm{H}^{\prime}=3,57$ bits ind $^{-1}$, mientras que el valor del índice de uniformidad o equidad de Pielou (J') fue de J'=0,61. El índice de diversidad calculado para las especies de Gastropoda fue de $\mathrm{H}^{\prime}=3,39$ bits ind $^{-1}$, mientras que el valor del índice de uniformidad o equidad de Pielou (J') fue de $\mathrm{J}^{\prime}=0,65$. Para Bivalvia de $\mathrm{H}^{\prime}=2,61$ bits ind $^{-1}$, mientras que el índice de Pielou (J') fue de J'=0,61 y para Polyplacophora fue de $\mathrm{H}^{\prime}=0,96$ bits ind $^{-1}$, mientras que el índice de Pielou (J') fue de $J^{\prime}=0,26$.

\section{Discusión}

Investigaciones sobre moluscos intermareales realizadas para el Estado de Guerrero como Flores (2004), donde se muestreó en 9 sitios de la costa Guerrerense, durante un ciclo anual, se reportaron 63 especies. La diferencia cuantitativa de riqueza de especies entre lo reportado por Flores (2004) y los resultados encontrados en el presente trabajo es mínima y esta diferencia principalmente se debe al esfuerzo de recolección de muestra, dado que Flores (2004) desarrolló los muestreos en varios sitios con diferentes tipos de sustrato rocoso existentes en la costa 
Tabla 3. Riqueza de especie. Familia, grado de aparición, densidad (organismos $\mathrm{m}^{-2}$ ) y longitudes (mm) de las especies de la Clase Polyplacophora asociadas al sustrato rocoso intermareal de playa Parque de la Reina, Acapulco, Guerrero, México / Species richness, Family, degree of species occurrence, density (organisms $\mathrm{m}^{-}$ $\left.{ }^{2}\right)$ and sizes $(\mathrm{mm})$ of the Class Polyplacophora species associated with intertidal rocky substrate at Parque de la Reina Beach, Acapulco, Guerrero, Mexico

\begin{tabular}{|c|c|c|c|c|c|c|c|}
\hline \multirow[b]{2}{*}{ Especies / Familias } & \multirow[b]{2}{*}{$\mathrm{n}$} & \multirow[b]{2}{*}{$\mathrm{Ga}$} & \multirow[b]{2}{*}{ De } & \multicolumn{4}{|c|}{ Longitud (mm) } \\
\hline & & & & Min. & Max. & Ave. & Ds \\
\hline \multicolumn{8}{|l|}{ ISCHNOCHITONIDAE } \\
\hline Ischnochiton (Ischnochiton) muscarius (Reeve, 1847) & 84 & $\mathrm{D}$ & 4,20 & 26,65 & 15,86 & 6,88 & 4,91 \\
\hline Stenoplax (Stenoplax) limaciformis (Sowerby, 1832) & 7 & $\mathrm{C}$ & 0,35 & 33,61 & 19,86 & 8,54 & 6,20 \\
\hline Lepidozona (Lepidozona) serrata (Carpenter, 1864) & 7 & $\mathrm{C}$ & 0,35 & 29,54 & 16,07 & 5,33 & 5,93 \\
\hline \multicolumn{8}{|l|}{ CALLISTOPLACIDAE } \\
\hline Callistochiton elenensis (Sowerby, 1832) & 8 & $\mathrm{C}$ & 0,40 & 30,97 & 18,01 & 5,11 & 4,57 \\
\hline Callistoplax retusa (Sowerby in Broderip \& Sowerby, 1832) & 8 & $\mathrm{C}$ & 0,40 & 4,35 & 4,35 & 4,35 & 0 \\
\hline \multicolumn{8}{|l|}{ CHAETOPLEURIDAE } \\
\hline Chaetopleura (Chaetopleur) lurida (Sowerby in Broderip \& Sowerby 1832) & 29 & $\mathrm{D}$ & 1,45 & 42.06 & 24,71 & 4,73 & 9,18 \\
\hline Chaetopleura (Chaetopleura) hanselmani (Ferreira, 1982) & 3 & $\mathrm{O}$ & 0,15 & 22,86 & 21,66 & 20,77 & 1,07 \\
\hline Chaetopleura (Chaetopleura) unilineata Leloup, 1954 & 10 & $\mathrm{O}$ & 0,50 & 13,77 & 10,52 & 6,26 & 3,12 \\
\hline \multicolumn{8}{|l|}{ CHITONIDAE } \\
\hline Chiton (Chiton) articulatus Sowerby, 1832 & 20 & $\mathrm{D}$ & 1,00 & 4,73 & 42,06 & 21,66 & 1,07 \\
\hline Chiton (Chiton) albolineatus Broderip \& Sowerby, 1829 & 1124 & $\mathrm{D}$ & 56,20 & 5,37 & 5,23 & 4,96 & 0,23 \\
\hline Tonicia forbesii forbesii Carpenter, 1857 & 3 & $\mathrm{O}$ & 0,15 & 8,54 & 33,61 & 19,86 & 6,20 \\
\hline \multicolumn{8}{|l|}{ TONICELLIDAE } \\
\hline Lepidochitona (Lepidochitona) salvadorensis (García-Ríos, 2006) & 5 & $\mathrm{O}$ & 0,25 & 22,85 & 13,25 & 5,04 & 5,47 \\
\hline Lepidochitona sp. 1 & 1 & $\mathrm{O}$ & 0,05 & 9,29 & 9,29 & 9,29 & 0 \\
\hline
\end{tabular}

$\mathrm{n}=$ número de organismos analizados; $\mathrm{Ga}=$ Grado de aparición $(\mathrm{D}=$ Dominante, $\mathrm{C}=\mathrm{Común}, \mathrm{NPF}=$ Numeroso poco frecuente, $\mathrm{O}=$ Ocasional); De=densidad; Ave=media, Ds=desviación típica

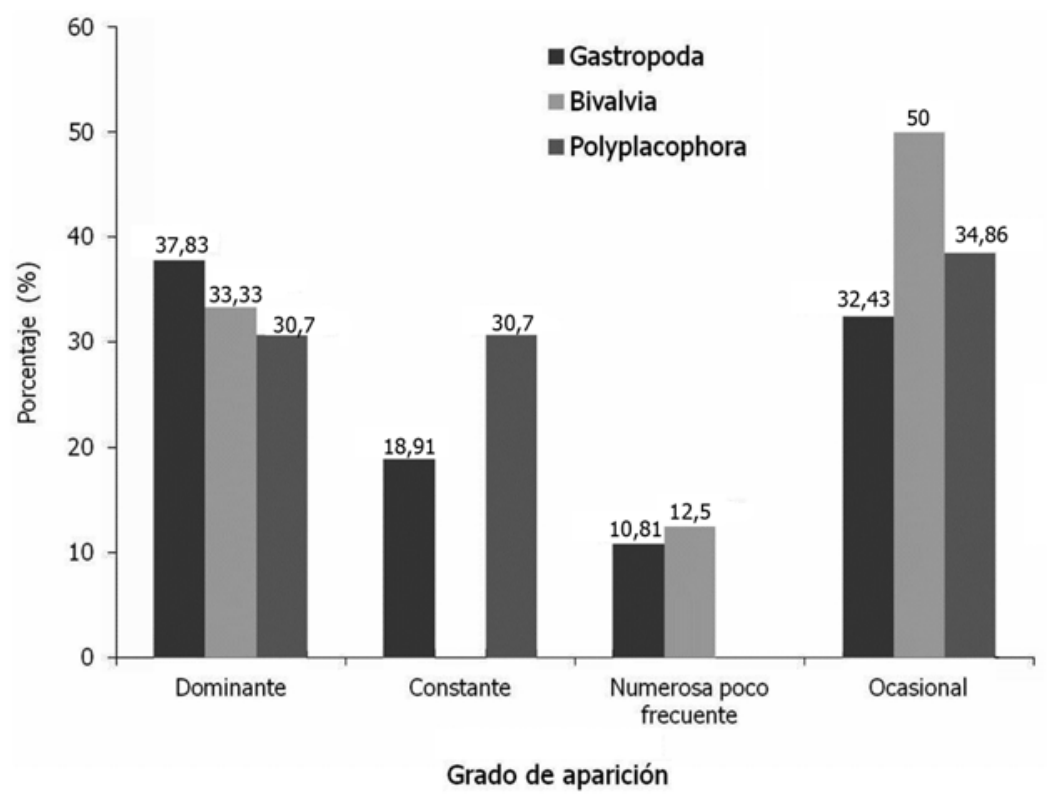

Figura 4. Grado de aparición de las especies de la comunidad de moluscos asociados al sustrato rocoso intermareal de la playa Parque de la Reina, Acapulco, Guerrero, México / Degree of species occurrence of mollusks community associated with intertidal rocky substrate at Parque de la Reina Beach, Acapulco, Guerrero, Mexico 
de Guerrero. En relación a las especies encontradas en la presente investigación coinciden 26 de las especies que reportó Flores (2004), sin embargo la mayoría de las especies reportadas por este autor no fueron encontradas en Parque de la Reina.

Otros trabajos llevados a cabo en playas rocosas ubicadas en la costa Guerrerense, pero fuera de la región marina prioritaria número 32 como los de Salcedo et al. (1988) en Zihuatanejo Guerrero, reportan 168 especies de moluscos, pero su trabajo lo realizan en los 3 niveles del intermareal, en todo tipo de sustrato, recolectando en 5 sitios, durante un año. Con este trabajo se coincide en el reporte de 36 especies.

Flores-Rodríguez et al. (2007) y Flores-Garza et al. (2007), trabajaron los moluscos del intermareal rocoso de Playa Troncones y Playa Ventura, respectivamente, determinando valores de riqueza de especies menores a lo que se encontró en la presente investigación. Con Flores-Rodríguez et al. (2007) coincide el reporte de 18 especies y con Flores-Garza et al. (2007) el reporte de 10 especies. La diferencia en el valor de la riqueza de especies, se debe en primer lugar a la estructura y complejidad de las playas, dado que los autores antes mencionados, desarrollaron sus muestreos en sitios cuyo sustrato se caracteriza por estar formado principalmente de macizos rocosos y de oleaje medio o intenso. Parque de la Reina es de acumulación (artificial) formado por bloques de granito y escombros de concreto introducidos por el hombre en el litoral lo que generan un medio más complejo, la intensidad del oleaje es baja, por lo que soporta una mayor riqueza de especies y diversidad. Esta relación entre riqueza, diversidad y complejidad del sustrato ya ha sido tratada por trabajos clásicos como el de Spight (1977), donde en sus generalizaciones sobre estudios de moluscos en costas rocosas tropicales y templadas, establece que el número de especies corresponde usualmente a la complejidad del hábitat y existe una relación entre diversidad y cualidades físicas del medio. Otro elemento a considerar es la metodología de colecta, dado que la unidad de muestreo fue cuidadosamente revisada y el tamaño de muestra es pequeño, además la playa es de fácil acceso y está protegida del oleaje intenso.

Estudios realizados sobre moluscos intermareales de la región marina prioritaria 32 como el de Flores-Garza et al. (2010) que se realizó en playa Tlacopanocha reporta una riqueza de 80 especies y se coincide en 43 especies. Flores-Garza et al. (2011), en un estudio realizado en Playa
Majahua encontraron mayor riqueza, reportando 99 especies, de las cuales se coincide en 47; cabe mencionar que los trabajos anteriores se llevaron a cabo con un metodología similar a la que se implementó en la presente investigación. La diferencia en el valor de la riqueza de especies encontrada por esta investigación y el trabajo antes mencionado, se debe a que la Playa Majahua es una playa de acumulación o de aluvión, caracterizada por cantos rodados de gran tamaño y macizos rocosos fijos, con presencia de fisuras y/o hendiduras, la intensidad del oleaje es muy baja, son playas muy protegidas, por lo tanto, la abrasión es mínima, con menor impacto antrópico. A diferencia de Parque de la Reina esta es una playa de acumulación artificial de rocas de tamaños variables, el oleaje es bajo a medio y el movimiento del sustrato rocoso que en su mayoría no es fijo causa una mayor abrasión, el impacto humano es mayor dado que se ubica a un costado del muelle del Puerto de Acapulco. Similarmente, Brusca (1980) en el Golfo de California, observó que playas compuestas de piedra muy grande son físicamente más estables que aquellas compuestas de pequeñas rocas o rocas sueltas y menciona la correlación directa entre estabilidad del hábitat y riqueza de especies. La diversidad de playas rocosas en término del número de especies presentes en este hábitat es mayor debido a su estabilidad y heterogeneidad. Por otra parte, Liñero-Arana \& Díaz (2006) en un estudio de poliquetos asociados a sustratos artificiales en Venezuela encontraron que las diferencias de las abundancias entre localidades podría atribuirse a las condiciones de cada localidad, aquellas que presentan mayor diversidad, densidad y riqueza se ubican en áreas relativamente tranquilas lo que facilita el asentamiento y colonización del substrato permitiendo, además, el desarrollo de una comunidad heterogénea y creando una mayor cantidad de microhábitats y por consiguiente una mayor diversidad y riqueza. Aldea \& Valdovinos (2005) en un análisis de moluscos intermareales del centro-sur de Chile menciona que la diversidad más baja en su estudio la reportó en los puertos con mayor grado de contaminación orgánica derivado de la industria pesquera.

Comparando las especies encontradas en las muestras analizadas por este estudio, con los listados existentes en otros reportes realizados en la región marina prioritaria 32, se encontraron 4 especies en Parque de la Reina para las que no existe registro. Con esto queda demostrado que falta conocimiento sobre los recursos faunístico marinos en el Estado de Guerrero. Por lo tanto, es muy importante disponer del inventario de especies, así como, 
conocer a las poblaciones y comunidades que componen la fauna marina de Acapulco y del Estado de Guerrero, ya que son la base para planificar, gestionar y conservar de forma responsable la diversidad biológica y hacer uso racional de los recursos marinos (Flores-Garza et al. 2011).

Este estudio también encontró a las familias Columbellidae de Gastropoda y a la Chitonidae de Polyplacophora como las familias mejor representadas por su abundancia. Se considera que las familias Muricidae, Columbellidae, Calyptraeidae, Isnochitonidae, Chitonidae, Chaetopleuridae y Mytilidae son las que representan a la comunidad de moluscos del intermareal rocoso de Parque de la Reina. No existe coincidencia en Polyplacophora y Bivalvia, donde Flores-Garza et al. (2011) reportan a la familia Tonicellidae y Chamidae como las mejor representadas.

En relación a la densidad de especies, García (1994), describe como las especies más importantes para Acapulco a Plicopurpura pansa (Gould, 1853), Mancinella triangularis, Nodilittorina modesta (Philippi, 1846), Siphonaria (Heterosiphonaria) palmata Carpenter, 1857, Siphonaria gigas Sowerby, 1825, Chama mexicana Carpenter, 1857, Chiton articulatus, mientras que Flores (2004), reporta a Nodilittorina aspera (Philippi, 1846), Plicopurpura pansa, M. triangularis, Petaloconchus complicatus Dall, 1908 y Brachidontes semilaevis (Menke, 1849) y Flores-Garza et al. (2011), reporta Crucibulum (Crucibulum) umbrella (Deshayes, 1830), Mancinella triangularis, Striostrea primatica (Gray, 1825.) Crassostrea palmula (Carpenter, 1857), Isognomon (Melina) janus (Carpenter, 1857), Chaetopleura (Chaetopleura) lurida (Sowerby in Broderip \& Sowerby 1832). Se coincide con Flores (2004) y Flores-Garza et al. (2011) en una especie, Mancinella triangularis, y con García (1994) se coincide con Chiton articulatus.

El valor estimado para la densidad de la comunidad de moluscos intermareales en playa Parque de la Reina, es el más alto que se ha reportado para el intermareal rocoso en playas de Guerrero. La diferencia en la densidad de especies con respecto a otros reportes hechos para el Estado de Guerrero, se debe, entre otros factores, al tipo y estabilidad del sustrato, lo que provoca una reducción en el número de especies, permaneciendo en la zona aquellas que tienen una mayor adaptación.

Referente a la composición de especies en la comunidad, se coincide con los reportes de Villalpando (1986), García (1994), Flores-Rodríguez et al. (2003, 2007), y Valdés-González et al. (2004) en que Mancinella triangularis, Nodilittorina aspera, Tectura fascicularis (Menke, 1851), Columbella fuscata Sowerby, 1832, Chiton articulatus, Mitrella ocellata (Gmelin, 1791), se presentaron como especies dominantes.

Por otra parte, se coincide con Flores-Garza et al. (2011), en que las especies dominantes y ocasionales fueron mayoritarias en la comunidad. Por lo tanto, se consideran como las representativas de la comunidad. Además, se coincide en que la Clase Polyplacophora presentan el mayor porcentaje de especies que se consideran representativas en la comunidad, ya que las especies dominantes y las comunes en conjunto suman 61,40\%. No existe coincidencia para las Clases Gastropoda y Bivalvia, ya que este estudio encontró a Gastropoda como la Clase que sigue de la Polyplacophora en cuanto al porcentaje de especies representativas de la comunidad y por último la Clase Bivalvia. Como lo mencionan FloresGarza et al. (2011) esto se debe a que Polyplacophora está mejor adaptado a las condiciones ambientales del intermareal rocoso, lo que se refleja en el diseño (plano, ovalado, valvas articuladas, faja y un gran pie muscular) de su cuerpo que es bastante eficiente para moverse y soportar las condiciones ambientales de la zona. Sin embargo la Clase Bivalvia se caracteriza fundamentalmente porque sus especies son sésiles, y en el caso de Parque de la Reina el sustrato es inestable y presenta mayor abrasión, lo que provoca una disminución en la densidad de las poblaciones y en el número de especies. La Clase Gastropoda presenta una gran cantidad de individuos móviles los cuales se pueden trasladar a lugares con sustrato más estable, con menor abrasión, donde encuentran protección, por eso este grupo se presentó como segundo lugar en cuanto a especies representativas.

En lo que respecta a los estadísticos descriptivos de la longitud de las especies representadas en las muestras, se coincide con Flores-Garza et al. (2011) en que Chama coralina y Chiton articulatus presentaron mayor longitud.

El índice de diversidad, así como el de equidad que se reporta en el presente trabajo es muy alto y corresponde a lo esperado en un sustrato rocoso, complejo, además es una zona tropical con escasas variaciones en la temperatura, lo que indica que Parque de la Reina es una zona muy diversa y con una alta uniformidad.

\section{Agradecimientos}

Agradecemos al Consejo Nacional de Ciencia y Tecnología y al Gobierno del Estado de Guerrero, a través 
del proyecto de investigación 91724 'Diversidad y abundancia de la cucaracha marina en las costas del Estado de Guerrero’ FOMIX 2008-01 y a la Universidad Autónoma de Guerrero, a través del proyecto de investigación 'Estudio de la biodiversidad y de la de la estructura comunidad de moluscos del intermareal rocoso de Acapulco, Guerrero (zona marina prioritaria 32) y establecimiento de la colección de moluscos en la UAEMUAGRO' por el financiamiento parcial para llevar a cabo este estudio. Gracias a Adriana Reyes Gómez por su colaboración en la determinación de las especies de Polyplacophora y a Arcadio Valdés González por sus observaciones y apoyo en la elaboración del manuscrito.

\section{LITERATURA CITADA}

Aldea C \& C Valdovinos. 2005. Moluscos del intermareal rocoso del centro-sur de Chile $\left(36^{\circ}\right.$ - $\left.38^{\circ} \mathrm{s}\right)$ : taxonomía y clave de identificación. Gayana 69(2): 364-396.

Arriaga L, E Vásquez, V Aguilar \& JM Espinoza 1998. Regiones prioritarias y planeación para la conservación de la biodiversidad, en Capital natural de México. Estado de conservación y tendencias de cambio, pp. 433-457. CONABIO, México.

Baqueiro E. 1979. Sobre la distribución de Megapitaria aurantiaca (Sowerby, 1831), M. squalida (Sowerby, 1835) y Dosinia ponderosa (Gray, 1838) en relación a la granulometría del sedimento (Bivalvia: Veneridae). Anales del Centro de Ciencias del Mar y Limnología, Universidad Nacional Autónoma de México 6: 25-32.

Baqueiro E \& J Stuardo. 1977. Observaciones sobre la biología, ecología y explotación de Megapitaria aurantiaca (Sowerby, 1831), M. squalida (Sowerby, 1835) y Dosinia ponderosa (Gray, 1838) (Bivalvia: Veneridae) de la bahía de Zihuatanejo e isla Ixtapa, Guerrero, México. Anales del Centro de Ciencias del Mar y Limnología, Universidad Nacional Autónoma de México 4: 161-208.

Barba-Marino F, P Flores-Rodríguez, R Flores-Garza, S García-Ibáñez \& DG Arana-Salvador. 2010. Biodiversidad y zonificación de la comunidad de moluscos, que habita el sustrato rocoso en dos sitios con distinta acción del oleaje, en la Isla «La Roqueta» Acapulco, Guerrero, México. En: Rangel LJ, J Gamboa, SL Arriaga \& WM Contreras (eds). Perspectiva en Malacología Mexicana, pp. 21-43. Universidad Juárez Autónoma de Tabasco, Villahermosa.

Brusca RR. 1980. Common intertidial invertebrates of the Gulf of California, 513 pp. The University of Arizona Press, Tucson.
Coan EV \& P Valentich-Scott. 2012. Bivalve seashells of tropical west America. Marine bivalve mollusks from Baja California to Peru. Monographs 6: 1-1258. Museum of Natural History, Santa Barbara.

Delgado VH. 1989. Estudio sistemático y aspectos ecológicos de gasterópodos de la facie rocosa de la bahía de Acapulco, Guerrero, México. Tesis de Licenciatura, Escuela Superior de Ecología Marina, Universidad Autónoma de Guerrero, Acapulco, 97 pp.

Esqueda MC, E Ríos-Jara, JE Michel-Morfín \& V LandaJaime. 2000. The vertical distribution and abundance of gastropods and bivalves from rocky beaches of Cuastecomate Bay, Jalisco. Revista de Biología Tropical 48: 765-775.

Flores P. 2004. Estructura de la comunidad de moluscos del mesolitoral superior en las playas de facie rocosa del Estado de Guerrero, México. Tesis doctoral, Facultad de Ciencias Biológicas, Universidad Autónoma de Nuevo León, San Nicolás de los Garzas, 207 pp.

Flores-Campaña LM, MA Ortiz-Arellano, JF ArzolaGonzález, FL González-Medina, E Cortez-Acosta \& D Rodríguez-García. 2007. Los quitones de las Islas de la Costa de de Sinaloa, México. En: Ríos-Jara E, MC EsquedaGonzález \& CM Galván-Villas (eds). Estudios sobre la Malacología y Conquiliología en México, pp. 42-44, Universidad de Guadalajara, Guadalajara.

Flores-Garza R, P Flores-Rodríguez, S García-Ibáñez \& A Valdés-González. 2007. Demografía del caracol Plicopurpura pansa (Neotaenioglossa: Muricidae) y constitución de la comunidad malacológica asociada en Guerrero, México. Revista de Biología Tropical 55(3-4): 867-887.

Flores-Garza R, C Torreblanca-Ramírez, P FloresRodríguez, S García-Ibáñez \& L Galeana-Rebolledo. 2010. Riqueza y análisis de la comunidad malacológica en el mesolitoral rocoso de la playa Tlacopanocha, Acapulco, Guerrero. En: Rangel LJ, J Gamboa, SL Arriaga \& WM Contreras (eds). Perspectiva en malacología mexicana, pp. 125-138. Universidad Juárez Autónoma de Tabasco, Villahermosa.

Flores-Garza R, C Torreblanca-Ramírez, P FloresRodríguez, S García-Ibáñez, L Galeana-Rebolledo, Valdés-González \& AA Rojas-Herrera 2011. Mollusc community from a rocky intertidal zone in Acapulco, Mexico. Biodiversity 12(3): 144-153.

Flores-Rodríguez P, R Flores-Garza, S García-Ibáñez \& A Valdés-González. 2003. Riqueza y diversidad de la malacofauna del mesolitoral rocoso de la Isla la Roqueta, Acapulco, Guerrero, México. Ciencia, Revista de Investigación Científica, Universidad Autónoma de Guerrero, México 11: 5-14. 
Flores-Rodríguez P, R Flores-Garza, S García-Ibáñez \& A Valdés-González. 2007. Variación en la diversidad malacológica del mesolitoral rocoso en Playa Troncones La Unión, Guerrero, México. Revista Mexicana de Biodiversidad 78: 33S-40S.

Flores-Rodríguez P, F Barba-Marino, R Flores-Garza, S García-Ibáñez \& DG Arana-Salvador. 2010. Análisis de la comunidad de moluscos del mesolitoral rocoso en playa Corralero, Oaxaca, México. En: Rangel LJ, J Gamboa, SL Arriaga \& WM Contreras (eds). Perspectiva en malacología mexicana, pp. 79-87. Universidad Juárez Autónoma de Tabasco, Villahermosa.

García JA. 1994. Fauna malacológica de acompañamiento del caracol Purpura pansa Gould 1853 en la zona mesolitoral de la isla Roqueta, Acapulco, Guerrero, México. Tesis Licenciatura, Escuela Superior de Ecología Marina, Universidad Autónoma de Guerrero, Acapulco, 97 pp.

García CI \& M Álvarez. 2007. Comunidades de quitones (Mollusca: Polyplacophora) de la Bahía de la Paz California Sur, México. Revista de Biología Tropical 55: 177-182.

Holguín OE \& AC González. 1989. Moluscos de la franja costera del Estado de Oaxaca, México, 221 pp. Dirección de Bibliotecas y Publicaciones, Instituto Politécnico Nacional, México.

Holguín-Quiñones OE \& AC González-Pedraza. 1994. Moluscos de la franja costera de Michoacán, Colima y Jalisco, México, 133 pp. Dirección de Bibliotecas y Publicaciones, Instituto Politécnico Nacional, México.

Kaas P \& RA Van Belle 1985. Monograph of living chitons (Mollusca: Polyplacophora). Suborder Ischnochitonina. Ischnochitonidae: Schizoplacinae, Callochitoninae \& Lepidochitoninae, 198 pp. E. J. Brill / Dr. W. Backhuys, Leiden.

Kaas P \& RA Van Belle 1990. Monograph of living Chitons (Mollusca: Polyplacophora). Suborder Ischnochitonina: Ischnochitonidae: Ischnochitoninae, 298 pp. E. J. Brill / W. Backhuys, Leiden.

Kaas P \& RA Van Belle 1994. Monograph of living chitons (Mollusca: Polyplacophora). Suborder Ischnochitonina. Ischnochitonidae: Ischnochitoninae (concluded), Callitoplacina; Mopaliidae, 402 pp. E. J. Brill / Dr. W. Backhuys, Leiden.

Keen AM. 1971. Sea shells of tropical West America, 1064 pp. Stanford University Press, California.

Landa-Jaime V \& J Arciniega-Flores. 1998. Macromoluscos bentónicos de fondos blandos de la plataforma continental de Jalisco y Colima, México. Ciencias Marinas 24: 155167.
Landa-Jaime V, M Cruz-Urzua, JE Michel-Morfín, J Archiniega-Flores, R Flores-Vargas \& C Amezcua. 2007. Guía ilustrada para la identificación de moluscos intermareales y de arrecifes en la Bahía de Tenacatita, Jalisco. En: Ríos-Jara E, MC Esqueda-González \& CM GalvánVillas (eds). Estudios sobre la Malacología y Conquiliología en México, pp. 62-64. Universidad de Guadalajara, Guadalajara.

Linero-Arana I \& DO Díaz. 2006. Poliquetos (Annelida: Polychaeta) epibiontes de Spondylus americanus (Bivalvia: Spondylidae) en el Parque Nacional Mochima, Venezuela. Revista de Biología Tropical 54(3): 765-772.

Olabarría C. 1999. Estructura y variación estacional de poblaciones de moluscos asociadas a la pesca artesanal de langosta en el Pacífico tropical. Revista de Biología Tropical 47: 851-865.

Ortíz-Arellano MA \& LM Flores-Campaña. 2008. Catálogo descriptivo e ilustrado de los moluscos de la zona intermareal de las islas de Navachiste, Sinaloa, México, 132 pp. Universidad Autónoma de Sinaloa y Gobierno del Estado de Sinaloa-Consejo Nacional de Ciencias y Tecnología, Mazatlán.

Reguero M \& A García-Cubas. 1989. Moluscos de la Plataforma Continental de Nayarit: Sistemática y ecología (cuatro campañas oceanográficas). Anales del Instituto de Ciencias del Mar y Limnología, Universidad Nacional Autónoma de México 16: 33-58.

Reyes-Gómez A, N Barrientos-Lujan, J Medina-Bautista \& S Ramírez-Luna. 2010. Chitons from the coralline area of Oaxaca, Mexico (Polyplacophora). Bollettino Malacologico 46: 111-125.

Ríos-Jara E, M Pérez-Peña, R Beas-Luna, E López-Uriarte \& E Juárez-Carrillo. 2001. Gastropods and bivalves of commercial interest from the continental shelf of Jalisco and Colima, México. Revista de Biología Tropical 49: 859863.

Román R, FM Cruz \& AL Ibáñez. 1991. Observaciones ecológicas de los moluscos de la zona intermareal de la bahía de Chamela, Jalisco, México. Anales del Instituto de Biología, Universidad Nacional Autónoma de México, Serie Zoología 62: 17-32.

Salcedo-Martínez S, G Green, A Gamboa \& P Gómez. 1988. Inventario de macroalgas y macroinvertebrados bénticos, presentes en áreas rocosas de la región de Zihuatanejo, Guerrero, México. Anales del Instituto Ciencias del Mar y Limnología, Universidad Nacional Autónoma de México 15: 73-96.

Sirenko B.2006. New outlook on the system of Chitons (Mollusca: Polyplacophora). Venus 65 (1-2): 27-49. 
Sokal RR \& FJ Rohlf. 1969. Biometry. The principles and practices of statistics in biological research, $776 \mathrm{pp}$. W. H. Freeman, San Francisco.

Skoglund C. 2001. Panamic province molluscan literature additions and changes from 1971 through 2000, I Bivalvia and II Polyplacophora, 139 pp. The Festivus, San Diego.

Skoglund C. 2002. Panamic province molluscan literature additions and changes from 1971 through 2001, III Gastropoda, 286 pp. The Festivus, San Diego.

Stuardo J \& M Villarroel. 1976. Aspectos ecológicos y distribución de los moluscos en las lagunas costeras de Guerrero, México. Anales del Centro de Ciencias del Mar y Limnología, Universidad Nacional Autónoma de México 3: 65-92.

Spight TM. 1977. Diversity of shallow-water gastropods communities on temperate and tropical beaches. American Naturalist 111: 1077-1097.
Taitt RV \& FA Dipper. 1998. Elements of marine ecology, 462 pp. Butterworth-Heinemann, Oxford.

Valdés-González A, P Flores-Rodríguez, R Flores-Garza \& S García-Ibáñez. 2004. Molluscan communities of rocky intertidal zone at two sites with different wave action on Isla la Roqueta, Acapulco, Guerrero, México. Journal of Shellfish Research 23: 875-880.

Villalpando E. 1986. Diversidad y zonación de moluscos de superficie rocosa, Isla Roqueta, Acapulco, Guerrero. Tesis de Licenciatura, Facultad de Ciencias, Universidad Nacional Autónoma de México, México, 150 pp.

Villarroel MM, A Magaña, B Gómez, O Del Río, J Lucio \& J Sánchez. 2000. Diversidad de moluscos en el litoral rocoso de Michoacán, México. Mexicoa 2: 54-63.

Recibido el 5 de marzo de 2012 y aceptado el 18 de mayo de 2012

Editor Asociado: Mauricio Landaeta D. 